\title{
Receiver Sensitivity Improvement for NRZ-OOK Signal by Optical Parametric Amplifier-Assisted Detection
}

\author{
Yu Liang, P. C. Chui, and Kenneth K. Y. Wong* \\ Photonic Systems Research Laboratory, Department of Electrical and Electronic Engineering, \\ The University of Hong Kong, Pokfulam Road, Hong Kong \\ *Email: kywong@eee.hku.hk
}

\begin{abstract}
By using a fiber optical parametric amplifier, we demonstrate a novel pre-amplification scheme to improve receiver sensitivity for $10-\mathrm{Gb} / \mathrm{s}$ non-return-to-zero on-off keying (NRZ-OOK) format with dual-end superposition of signal and idler. We achieve receiver sensitivity of -40.5 $\mathrm{dBm}$, and improve by $2 \mathrm{~dB}$ when comparing to its single-end counterpart. (C)2009 Optical Society of America

OCIS codes: (060.2320) Fiber optics amplifiers and oscillators; (190.4410) Nonlinear optics, parametric processes.
\end{abstract}

\section{Introduction}

Fiber-based optical parametric amplifier (OPA) with femtosecond response time [1], high gain [2] and wide gain bandwidth [3], has been shown to have comparable performance with conventional optical amplifiers [4]. Recently a novel pre-amplifier has been demonstrated for return-to-zero on-off keying (RZ-OOK) signal [5]. However, NRZOOK is also a key format with narrow bandwidth in current systems. In order to comprehensively study the preamplifier based on OPA for practical amplification, it is necessary to investigate performance of other formats. Therefore, in this paper, we investigate receiver sensitivity improvement for 10-Gb/s NRZ-OOK signal based on the novel OPA-assisted detection scheme.

\section{Principle}

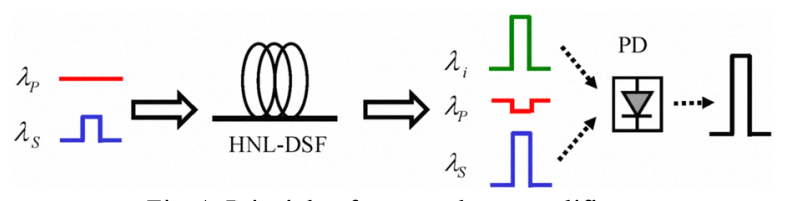

Fig. 1. Principle of proposed pre-amplifier.

The principle of pre-amplifier is shown in Fig. 1. The inputs to the nonlinear medium are weak NRZ-OOK signal with wavelength $\lambda_{S}$ and a strong CW pump with wavelength $\lambda_{P}$. When parametric amplification occurs, the pattern on signal can be transcribed to idler together with pump depletion. By launching both the amplified signal and idler into a photodetector (PD), the output current of PD will be constructively superimposed. Therefore, an output electrical signal with higher voltage swing is obtained.

\section{Experiment}

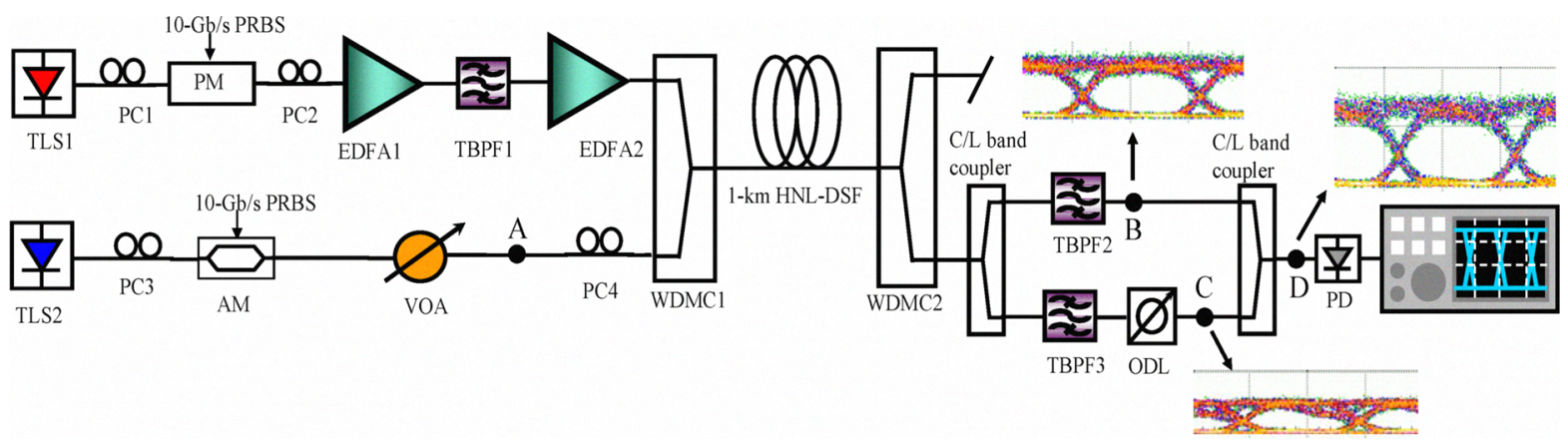

Fig. 2. Experimental setup. Insets are eye diagrams of $10-\mathrm{Gb} / \mathrm{s}$ signal, idler, and combination at point $\mathrm{B}, \mathrm{C}$, and $\mathrm{D}$, respectively. Time base: $50 \mathrm{ps} / \mathrm{div}$.

The experimental setup is shown in Fig. 2. The parametric gain medium consist of a spool of 1-km highly-nonlinear dispersion-shifted fiber (HNL-DSF) with nonlinear coefficient $\gamma \approx 14 \mathrm{~W}^{-1} \mathrm{~km}^{-1}$ and zero-dispersion wavelength $\lambda_{0} \approx$ $1560 \mathrm{~nm}$. The OPA pump is supplied from a tunable laser source (TLS1) set at $1561.1 \mathrm{~nm}$. It is phase-modulated with $10-\mathrm{Gb} / \mathrm{s} 2^{31}-1$ pseudo-random binary sequence (PRBS) for stimulated Brillouin scattering (SBS) suppression. The phase-dithered pump goes through two stage erbium-doped fiber amplifiers (EDFAs) to be amplified to 27.7 


\section{TuY4.pdf}
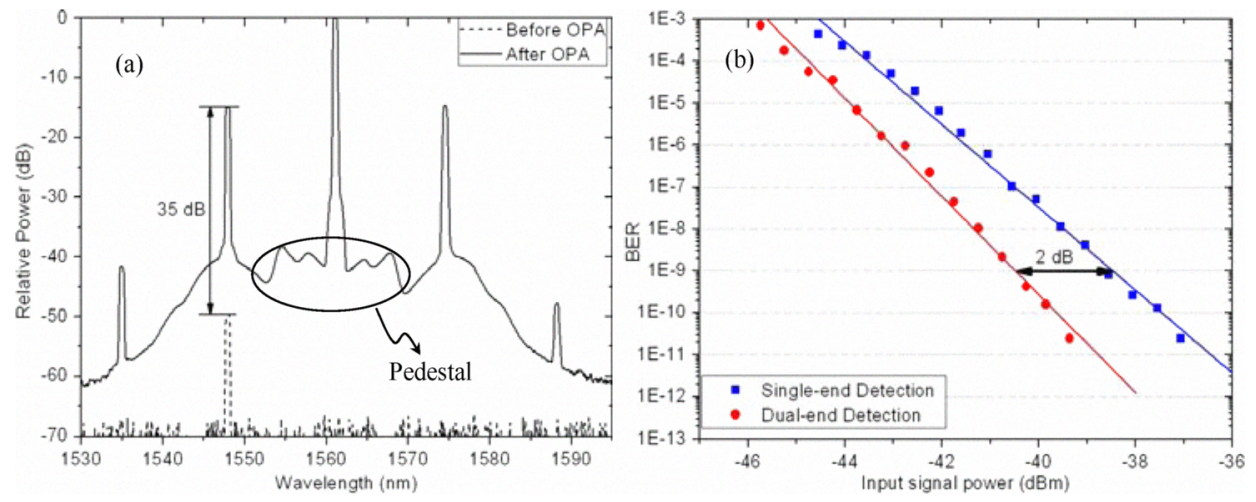

Fig. 3. (a) Spectra before and after OPA at the output of HNL-DSF; (b) BER plots of single-end detection and dual-end detection.

$\mathrm{dBm}$. A tunable band-pass filter (TBPF1) between them is used to reduce the amplification spontaneous emission (ASE) noise at the input of EDFA2. The 10-Gb/s NRZ-OOK is generated by intensity-modulating lightwave from TLS2 at $1548.0 \mathrm{~nm}$ by a Mach-Zehnder intensity modulator (MZ-IM) with 10-Gb/s $2^{31}-1$ PRBS. The pump and signal are combined using a WDM coupler (WDMC1) and launched into HNL-DSF for parametric amplification. Maximum OPA gain of $35 \mathrm{~dB}$ is attained by aligning the state of polarizations (SOPs) of signal and pump through polarization controllers PC2 and PC4. Then the pump is filtered out by WDMC2. The signal and idler are split by a $\mathrm{C} / \mathrm{L}$ band coupler and then filtered by TBPF2 and TBPF3. And the path length is compensated by an optical delay line (ODL). They are combined again using another $\mathrm{C} / \mathrm{L}$ band coupler. The output is monitored through digital communication analyzer (DCA) with 30-GHz PD and bit-error rate tester (BERT) with 10-GHz PD. The sensitivity of the receiver is defined as the signal power measured at the input port of WDMC1 (denoted as point A in Fig. 2), which is controlled by a variable optical attenuator (VOA). The eye diagram at point B is the amplified signal and the one at point $\mathrm{C}$ is the idler. The amplitude of idler is lower than that of signal in this experiment because the $\mathrm{L}$ band filter (TBPF3) in idler branch has higher insertion loss than C-band filter (TBPF2) in signal branch. The better noise suppression on mark level of idler than that of signal owes to the 0.2-nm bandwidth TBPF3 compared to 0.8$\mathrm{nm}$ bandwidth TBPF2. The distortion on mark level of idler is due to phase-modulation to intensity-modulation (PM-IM) conversion induced by SBS suppression [6]. After combining the signal and idler, it can be observed that the eye opening of the dual-detection scheme enjoy wider eye opening due to its constructive superposition of signal and idler.

Fig. 3 (a) shows the spectra at the output of HNL-DSF, where 35-dB internal parametric gain can be obtained. Note that the pedestal of pump is caused by the WDMC1. BER of single- and dual-end detection schemes are measured to quantify the benefit of dual-end detection. At BER of $10^{-9}$, receiver sensitivity of dual-end detection reaches down to $-40.5 \mathrm{dBm}$. Compared to single-end scheme, this dual-end scheme is improved by $2 \mathrm{~dB}$. Such improvement is contributed by the increase in signal swing from the idler.

\section{Conclusion}

Receiver sensitivity improvement technique for $10-\mathrm{Gb} / \mathrm{s}$ NRZ-OOK signal has been demonstrated using constructive superposition of signal and idler in OPA by a dual-end detection scheme. Low receiver sensitivity can approach $-40.5 \mathrm{dBm}$ and power penalty was improved by $2 \mathrm{~dB}$ compared to single-end detection scheme. The configuration of this pre-amplifier indicate that it can be extend to WDM systems and useful in OPA-assisted systems.

\section{Acknowledgment}

The work was partially supported by a grant from the Research Grants Council of the Hong Kong Special Administrative Region, China (Projects No. HKU 7172/07E and HKU 7179/08E). The authors would also like to acknowledge Sumitomo Electric Industries for providing the HNL-DSF.

\section{References}

[1] G. P. Agrawal, Nonlinear fiber optics (Academic Press, 2001), Chap. 10.

[2] T. Torounidis, P. A. Andrekson and B. E. Olsson, "Fiber-optical parametric amplifier with 70-dB gain," IEEE Photon. Technol. Lett., vol. 18, no. 10, pp. 1194-1196, 2006.

[3] M. E. Marhic, K. K. Y. Wong and L. G. Kazovsky, "Wide-band tuning of the gain spectrum of one-pump fiber optical parametric amplifiers," IEEE J. Select. Topics Quantum Electron., vol. 10, no. 5. pp. 1133-1141, 2004.

[4] J. Hansryd and P. A. Andrekson, "Broad-band continuous-wave-pumped Fiber Optical Parametric Amplifier with 49-dB Gain and Wavelength -Conversion Efficiency," IEEE Photon. Technol. Lett., vol. 13, no. 3, pp. 194-196, 2001.

[5] Y Liang, P. C. Chui, and K. K. Y Wong, "A Novel Pre-amplifier Based on Fiber Optical Parametric Amplifier," accepted by OECC 2009.

[6] K. K. Y Wong, M. E. Marhic, and L. G. Kazovsky, "Phase-Conjugate Pump Dithering for High-Quality Idler Generation in a Fiber Optical Parametric Amplifier," IEEE Photon. Tech. Lett., vol. 15, no. 1, pp. 33-35, 2003. 